\title{
Non-Absorption of Ingested Lipophilic and Amphiphilic Allelochemicals by Generalist Grasshoppers: The Role of Extractive Ultrafiltration by the Peritrophic Envelope
}

\author{
Raymond V. Barbehenn
}

\author{
Department of Biology, University of Michigan, Ann Arbor, Michigan
}

The role of the peritrophic envelope in the non-absorption of three allelochemicals ingested by generalist grasshoppers was examined. This study tested the hypothesis that the association of lipophilic and amphiphilic allelochemicals with lipid aggregates (mixed micelles) reduces their permeability through the peritrophic envelope, a process similar to extractive ultrafiltration. Each of three allelochemicals (digitoxin, ouabain, and xanthotoxin) were solubilized in a lysolecithin suspension and injected separately into the midgut lumens of adult Melanoplus sanguinipes (Orthoptera: Acrididae). The low permeability of digitoxin through the peritrophic envelope was consistent with the extractive ultrafiltration of this compound. By comparison, ouabain and xanthotoxin permeability coefficients were 7- and 12-fold higher, respectively, than those of digitoxin. The results of extractive ultrafiltration assays confirmed that digitoxin is effectively extracted in lysolecithin micelles, but that neither ouabain nor xanthotoxin aggregates efficiently with these micelles. Arch. Insect Biochem. Physiol. 42:130-137, 1999. ๑ 1999 Wiley-Liss, Inc.

Key words: Melanoplus sanguinipes; Orthoptera; Acrididae; peritrophic membrane.

\section{INTRODUCTION}

Previous work has shown that generalist grasshoppers can tolerate certain ingested plant allelochemicals by non-absorption. In particular, Melanoplus sanguinipes absorbs little or no digitoxin (Smirle and Isman, 1992), sesquiterpene lactones (Isman, 1985), $\alpha$-terthienyl or xanthotoxin (Berenbaum and Isman, 1989). A study of another generalist grasshopper, Schistocerca gregaria, has also shown that it is protected from absorbing digitoxin and ouabain (Scudder and Meredith, 1982). Non-absorption of allelochemicals might result from the impermeability of the peritrophic envelope, the midgut epithelium, or both struc- tures. However, none of the previous studies has distinguished between these possibilities.

The peritrophic envelope is a thin secreted tubular structure, composed of one or more peritrophic membranes, that surrounds all food in the midguts of insects. The peritrophic envelope has often been likened to an ultrafilter (e.g.,

Contract grant sponsor: NSF; Contract grant numbers IBN94-07128, IBN-96-03621.

*Correspondence to: Raymond V. Barbehenn, University of Michigan, Department of Biology, Ann Arbor, MI 48109-1048. E-mail: rvb@umich.edu

Received 3 March 1999; accepted 18 June 1999. 
Zhuzhikov, 1970; Terra and Ferreira, 1981; Peters and Wiese, 1986; Miller and Lehane, 1990). Factors limiting the permeability of allelochemicals through this structure include size and possibly charge (Miller and Lehane, 1993; Barbehenn and Martin, 1995, 1997, 1998). Factors limiting the absorption of allelochemicals through the midgut epithelium include properties of the chemicals that influence their solubility in phospholipid membranes (polarity, size, and charge) (Shaw and Guthrie, 1970; Duffey, 1980), the efficiency of the detoxification systems in the midgut epithelium (Lindroth, 1991), and the rate of passage from the midgut epithelium to the hemocoel.

One potential means by which the peritrophic envelope could contribute to the process of allelochemical non-absorption is by the retention of allelochemicals sequestered in lipid aggregates in the gut fluid of herbivorous insects. Lipophilic and amphiphilic allelochemicals would be expected to partition into the lipid phase of gut fluid in inverse proportion to their water solubility (Florence, 1977; Kuksis, 1987). If these aggregates are sufficiently large, then allelochemical absorption could be impeded by a process similar to extractive ultrafiltration. In this separation process lipophilic and amphiphilic compounds are extracted into a non-aqueous phase that does not permeate an ultrafilter (Watters et al., 1989). The non-aqueous phase of the midgut fluid in herbivorous insects is composed primarily of micelles (Martin and Martin, 1984; Turunen and Chippendale, 1989; Small, 1986). Micelles form during the digestive process from the aggregation of lysophospholipids (surfactants), galactosyl glycerides, long chain fatty acids, and other amphiphilic and lipophilic compounds (Hawke, 1973; Turunen and Chippendale, 1989). Although pure surfactant micelles range in diameter from 3 to $12 \mathrm{~nm}$, mixed micelles may be hundreds of nanometers in diameter (Vander et al., 1980; Mimms et al., 1981; Furth et al., 1984; Jones et al., 1987). Emulsified lipid droplets are also relatively large $(\geq 1,000 \mathrm{~nm})$ (Masoro, 1968; Vander et al., 1980), but these are a minor component of the midgut fluid of foliagefeeding insects (Hawke, 1973; Turunen and Chippendale, 1989). Maximum particle sizes that permeate the matrix of the peritrophic membranes of herbivorous insects have been estimated to range from $7.0-8.0 \mathrm{~nm}$, based on digestive en- zyme permeability (Santos and Terra, 1986; Ferreira et al., 1994), to 21-36 nm, based on FITC-dextran permeability (Barbehenn and Martin, 1995). Therefore, depending on the size limitation of pores in the matrix and the sizes of lipid-allelochemical aggregates, the association of allelochemicals with lipid aggregates might reduce allelochemical permeation, either by size exclusion or reduced rates of diffusion.

The allelochemicals examined in this study have a wide range of water solubilities: ouabain is a polar cardiac glycoside with a maximum solubility of $23 \mathrm{mM}$; digitoxin is a nonpolar cardiac glycoside with a maximum solubility of $0.013 \mathrm{mM}$; and xanthotoxin is a furanocoumarin that is "practically insoluble" (Merck, 1996). In this study the degree of association between each of these allelochemicals and lysolecithin micelles was determined using extractive ultrafiltration. To examine the potential of the peritrophic envelope to retain allelochemicals in mixed micelles, each of the three allelochemicals was solubilized in a lysolecithin suspension and injected separately into the midgut lumens of adult $M$. sanguinipes. The rates of permeation of these allelochemicals from two types of midgut preparations were compared. One preparation contained an intact peritrophic envelope surrounded by a midgut wall. A small hole was cut to expose the peritrophic envelope, thereby allowing the relative permeability of the peritrophic envelope to be measured for allelochemicals that do not permeate the midgut wall. A second type of midgut preparation, in which the wall remained intact but the peritrophic envelope was removed, was made to measure the permeability of the midgut wall. The results of these studies were considered consistent with extractive ultrafiltration if the permeability of the allelochemical through the peritrophic envelope is low and if the allelochemical is efficiently retained along with lysolecithin micelles in an extractive ultrafiltration assay.

\section{MATERIALS AND METHODS Chemicals}

Digitoxin, xanthotoxin, and lysolecithin (soy and palmitoyl) were purchased from Sigma Chemical Co. (St. Louis, MO). Soy lysolecithin (lysophosphatidylcholine) is a mixture of fatty acid 
esters, including linoleoyl (48.5\%), palmitoyl (25\%), stearoyl (8\%), and linolenoyl (4.5\%). Ouabain was purchased from Fluka Chemical Co. (Ronkonkoma, NY). All chemicals were weighed to the nearest $0.01 \mathrm{mg}$ on a Cahn Electrobalance.

\section{Insects}

Eggs of $M$. sanguinipes (Orthoptera: Acrididae) were obtained from the USDA (Bozeman, MT). A colony was maintained in a screen cage $(1 \mathrm{~m} \times 0.5 \mathrm{~m} \times 0.5 \mathrm{~m})$ with a $150 \mathrm{~W}$ light bulb placed against the screen to provide a heat gradient (16 h L:8 h D). Nighttime temperature was ambient (ca. $22^{\circ} \mathrm{C}$ ). Insects were reared from egghatch on romaine lettuce (Lactuca sativa longifolia) and wheat bran. Adult insects were used for all experiments.

\section{Peritrophic Envelope and Midgut Wall Permeability}

A separate experiment was performed for each allelochemical and midgut preparation type. Allelochemical solutions or suspensions were prepared by sonicating the allelochemical $(20 \mathrm{~min}$, $22^{\circ} \mathrm{C}$ ) in an aqueous suspension of palmitoyl lysolecithin (41-71 mM). Each insect was chilled $\left(-20^{\circ} \mathrm{C}, 9 \mathrm{~min}\right)$ and its entire gut was dissected free from the body wall under magnification from a dissecting microscope. The gut was placed in a small dish of incubating solution containing $5 \mathrm{mM}$ Tris(hydroxymethyl)aminomethane, $45 \mathrm{mM}$ Tris(hydroxymethyl)aminomethane hydrochloride, 20 $\mathrm{mM} \mathrm{CaCl} 2,3 \mathrm{mM} \mathrm{NaCl}, 227 \mathrm{mM} \mathrm{KCl}, 41 \mathrm{mM}$ $\mathrm{MgSO}_{4}, 6 \mathrm{mM}$ ascorbic acid, and $440 \mathrm{mM}$ fructose $(\mathrm{pH}$ 7.0). The foregut and anterior midgut (including the caeca) were removed, and the hindgut was ligated adjacent to the midgut with a silk suture (size 6-0). Allelochemicals were injected into the midgut lumen to allow large doses to be introduced rapidly. A Hamilton microsyringe (10 $\mathrm{ml}$ ) containing an allelochemical solution or water (control) was mounted in a micromanipulator, and the gut preparation was tied onto the needle tip while remaining in the incubating solution. A $1.5 \mu \mathrm{l}$ aliquot of a test solution was introduced into the closed endoperitrophic space. The gut preparation was pulled from the needle tip with tension on the suture ends to prevent the introduced solution from leaking. In gut preparations containing an intact peritrophic en- velope, a small hole was cut in the mid-midgut wall to expose the peritrophic envelope. Damage to the peritrophic envelope was monitored by carefully observing any outflow of gut fluid into the incubating solution during dissection, and by examing the data for statistical outliers (Barbehenn and Martin, 1995; Barbehenn et al., 1996). Areas $\left(\mathrm{mm}^{2}\right)$ of holes made in experiments on the permeability of digitoxin, ouabain, and xanthotoxin averaged $1.8( \pm 0.3 \mathrm{SE}), 1.1( \pm 0.2)$, and $0.9( \pm 0.1)$, respectively. After rinsing each gut preparation for approximately $10 \mathrm{sec}$ in each of three beakers of incubating solution, they were incubated for $1.5 \mathrm{~h}$ at $22^{\circ} \mathrm{C}\left( \pm 1^{\circ}\right)$ in $1.5 \mathrm{ml}$ of incubating solution containing palmitoyl lysolecithin (2.0 mM; pH 7.0). Following the incubation period, the entire volume of incubating solution was placed in a test tube, frozen in an ethanoldry ice bath, and lyophilized. To determine the amount of allelochemical remaining in the midgut following the incubation period, the midgut contents were extracted in methanol $(2 \times 1.0 \mathrm{ml}$, $1 \mathrm{hr}$ ). The percentage of each allelochemical recovered at the end of the incubation period ( $[\mu \mathrm{g}$ remaining in lumen $+\mu \mathrm{g}$ present in incubating solution $] / \mu \mathrm{g}$ injected) was $102.2 \%$ (digitoxin), $77.8 \%$ (ouabain), and $81.8 \%$ (xanthotoxin).

To determine the role of the midgut wall in limiting the permeation of test chemicals from gut preparations, the above procedures were repeated with the following exceptions: the peritrophic envelope and midgut contents were removed and the midgut wall remained intact. The efficiency of recovery of allelochemicals introduced into the midgut lumen was assumed to be similar to that measured from the entire midgut.

\section{HPLC Analysis}

Allelochemical concentrations in solutions and suspensions injected into the midgut lumen were measured in replicate aliquots $(2,4$, or $5 \mu \mathrm{l})$ dissolved in 0.5 or $1.0 \mathrm{ml}$ of methanol. Lyophilized incubating solutions were resolubilized sequentially in $200 \mu \mathrm{l}$ of double-distilled water and 600 $\mu \mathrm{l}$ of methanol, and filtered $(0.45 \mu \mathrm{m}$, Gelman GHP Acrodisc). Aliquots (10-30 $\mu \mathrm{l})$ of samples and standards were injected onto a Vydac C-18 column $(250 \times 4.6 \mathrm{~mm}, 5 \mu)$ and guard column using a Shimadzu autoinjector. Digitoxin and xanthotoxin were eluted at $1.0 \mathrm{ml} / \mathrm{min}$ using the fol- 
lowing gradient: $0-15 \mathrm{~min}: 28 \%$ to $90 \%$ acetonitrile; $15-25$ min: $90 \%$ to $28 \%$ acetonitrile; and 30 45 min: $28 \%$ acetonitrile. Ouabain was measured using the following gradient elution profile (1.0 $\mathrm{ml} / \mathrm{min}$ ): $0-15 \mathrm{~min}: 18 \%$ to $70 \%$ acetonitrile; $15-$ $20 \mathrm{~min}: 70 \%$ to $18 \%$ acetonitrile; and $20-40 \mathrm{~min}$ : $18 \%$ acetonitrile. All compounds were measured with a Shimadzu SPD-6AV u.v.-visible detector (0.002 AUFS). Ouabain was detected at $220 \mathrm{~nm}$, xanthotoxin at $249 \mathrm{~nm}$, digitoxin at $240 \mathrm{~nm}$, and soy lysolecithin at each wavelength. Peak areas were integrated with a Shimadzu C-R4A Chromatopac integrator, and converted to $\mu$ g injected with standard curves for each compound. Average peak areas of interfering substances in control samples, when present, were subtracted from the peak areas in treatment samples.

\section{Extractive Ultrafiltration}

Suspensions of digitoxin, ouabain, and xanthotoxin were sonicated $(20 \mathrm{~min})$ in soy lysolecithin $(1 \mathrm{mg} / \mathrm{ml})$ in double-distilled water. Soy lysolecithin was substituted for palmitoyl lysolecithin to allow measurement with a u.v.-visible detector. Micellar suspensions were centrifuged $\left(13,600 \mathrm{~g}, 10 \mathrm{~min}, 21^{\circ} \mathrm{C}\right)$ and filtered $(0.45 \mu \mathrm{m})$. Replicate aliquots $(350$ or $400 \mu \mathrm{l})$ were placed in ultrafiltration devices (Centrifree MPS-1; Amicon) containing 30,000 molecular weight cutoff (MWCO) ultrafilter membranes. Ultrafiltration devices were centrifuged (Sorvall RC-5B, 500g, 8 or $10 \mathrm{~min}$ ), and the volumes of the ultrafiltrate $(<30 \mathrm{kDa}$ MWCO) and the retentate were measured. Concentrations of allelochemicals and lysolecithin in these fractions and in the unfiltered solutions were measured with HPLC, as described above.

\section{Xanthotoxin Solubility}

The putative insolubility of xanthotoxin in water was tested by sonicating $0.47 \mathrm{mg}$ of xanthotoxin in double-distilled water $(4.73 \mathrm{ml}, 20$ $\min )$, and centrifuging the suspension $(13,600 \mathrm{~g}$, $\left.4 \mathrm{~min}, 21^{\circ} \mathrm{C}\right)$. The supernatant was prefiltered $(0.45 \mu \mathrm{m})$, and an aliquot $(500 \mu \mathrm{l})$ was filtered through a 3000 MWCO ultrafilter (Microcon microconcentrator; Amicon) by centrifugation $\left(13,600 \mathrm{~g}, 15 \mathrm{~min}, 21^{\circ} \mathrm{C}\right)$. The concentrations of xanthotoxin in the supernatant suspension and in the ultrafiltrate were measured with HPLC, as described above.

\section{Micelle Size}

Micelle size was measured with a Coulter N4 Plus submicron particle size analyzer. Particle size measurements were made with the size distribution program at 62.6 or $90.0^{\circ}$ light-scatter angles. Soy lysolecithin $(10.0 \mathrm{mg} / \mathrm{ml})$ or palmitoyl lysolecithin $(11.4 \mathrm{mg} / \mathrm{ml})$ were solubilized in 10 mM HEPES buffer ( $\mathrm{pH} 7.4$ ) containing $0.145 \mathrm{M}$ $\mathrm{NaCl}$. The effect of digitoxin on micelle size was measured by examining micelle size in a soy lysolecithin solution $(17.2 \mathrm{mg} / \mathrm{ml})$ in HEPES buffer and remeasuring micelle size after solubilizing digitoxin with sonication $(1.1 \mathrm{mM}$ final concentration). Xanthotoxin particle size was measured in double-distilled water, prepared as described above $(0.096 \mathrm{mM}$ final concentration). All solutions were centrifuged $\left(13,600 \mathrm{~g}, 5 \mathrm{~min}, 21^{\circ} \mathrm{C}\right)$ and filtered $(0.45 \mu \mathrm{m})$ to remove insoluble particles. Three to eleven replicates of each preparation were measured.

\section{Statististical Analyses and Calculations}

Pairwise comparisons of means were made using Mann-Whitney U-tests (SYSTAT; Wilkinson, 1990). Correlations between hole diameter in midgut preparations and percent permeation of each allelochemical were examined (SYSTAT; Wilkinson, 1990). No significant correlations were found in individual experiments or in pooled data and, therefore, hole diameter was not used in the calculation of permeability coefficients. The permeability of a gut preparation $(\mathrm{P})$ was calculated from the equation $\mathrm{P}=\mathrm{p} / \mathrm{Ct}$, where $\mathrm{p}$ is the total number of mmoles of the test chemical that diffused into the incubating solution in time $\mathrm{t}(\mathrm{hr})$ and $\mathrm{C}$ is the concentration $\left(\mu \mathrm{moles} / \mathrm{cm}^{3}\right)$ of the test chemical initially present in the midgut. Using this formula, $\mathrm{P}$ has the units $\mathrm{cm}^{3} / \mathrm{h}$. The average volumes of the sausage-shaped gut preparations with and without peritrophic envelopes ( $n=5$ each) was $6 \mu \mathrm{l}$. Volumes were measured by placing midgut preparations (with the sutures and underlying tissue removed) in a microcentrifuge tube containing $50 \mu \mathrm{l}$ of water, and homogenizing the contents with a spatula tip. The total volume was measured with an adjustable pipet (Gilson). The midgut volume was calculated as the total volume minus the original volume. Assuming that the surface areas of similar shapes with similar volumes are essentially the same, the permeabil- 
ity coefficients can be compared between gut preparations. Back-diffusion of allelochemicals into midgut preparations was calculated to be negligible.

\section{RESULTS}

Permeability coefficients from midgut preparations that contained an intact peritrophic envelope were reduced $27 \%$ for digitoxin, $64 \%$ for ouabain, and $36 \%$ for xanthotoxin, compared with preparations from which the peritrophic envelope had been removed (Table 1), although only the reduced permeability of ouabain was statistically significant $(P=0.01)$. Midgut preparations, with or without an intact peritrophic envelope, were much more permeable to ouabain and xanthotoxin than to digitoxin (Table 1). The permeability coefficients for ouabain and xanthotoxin are 14.5 and 14.0 times higher than the permeability coefficient for digitoxin in gut preparations without a peritrophic envelope and 7.2 and 12.3 times higher in gut preparations with a peritrophic envelope.

The large percentage of ouabain and xanthotoxin that diffused from the midgut preparations is less than would be predicted at equilibrium. Had equilibrium been reached during the $1.5 \mathrm{~h}$ incubation period, over $99 \%$ of the allelochemicals would have been in the incubation solutions, since the average volume of the $M$. sanguinipes gut preparations was $6 \mu \mathrm{l}$ and the volume of the incubation solutions was $1.5 \mathrm{ml}$.

Lysolecithin micelles do not pass through an ultrafiltration membrane with a MWCO of 30,000 (Table 2). The concentration of lysolecithin in ultrafiltrates was only 1-4\% of its concentration in the original suspensions. When digitoxin was added to a lysolecithin suspension, it was also extracted by ultrafiltration. The concentration of digitoxin in the ultrafiltrate was only $14 \%$ of its concentration in the original suspension (Table 2). By contrast, ultrafiltration does not result in the concentration of ouabain in a lysolecithin suspension (Table 2). These results suggest that the two cardiac glycosides have markedly different abilities to form mixed micelles. Xanthotoxin in a lysolecithin suspension is moderately concentrated by ultrafiltration. The concentration of xanthotoxin in the ultrafiltrate was $63 \%$ of its concentration in the original solution, suggesting that a substantial fraction of the xanthotoxin was not associated with lysolecithin micelles (Table 2). This result is consistent with the observation that the concentration of xanthotoxin in double-distilled water was between $52-59 \%$ of its concentration in lysolecithin suspensions. Further ultrafiltration (3000 MWCO) had no effect on the concentration of xanthotoxin in double-distilled water. These suspensions contained $1.0 \pm 0.2 \mathrm{~nm}$ diameter particles. By contrast, soy and palmitoyl lysolecithin micelles had diameters of $8.3 \pm 0.3$ and $8.0 \pm 0.06 \mathrm{~nm}$, respectively. Soy lysolecithin micelles in suspensions with and without digitoxin measured $7.3 \pm 0.1 \mathrm{~nm}$ and $7.6 \pm 0.2 \mathrm{~nm}$, respectively.

\section{DISCUSSION}

The results of this study are consistent with the extractive ultrafiltration of digitoxin by the peritrophic envelope, i.e., digitoxin aggregates in lysolecithin micelles and has very low permeability coefficients through the peritrophic envelope in $M$. sanguinipes. Therefore, non-absorption of digitoxin by $M$. sanguinipes (Smirle and Isman,

TABLE 1. Permeability of Melanoplus sanguinipes Midgut Preparations to Allelochemicals*

\begin{tabular}{llcrrr}
\hline Compound (N) & $\begin{array}{c}\text { Type of gut } \\
\text { preparation }\end{array}$ & $\begin{array}{c}\text { Concentration } \\
\text { in midgut } \\
\left(\mu \text { moles cm }^{-3}\right)\end{array}$ & $\begin{array}{c}\text { Amount } \\
\text { permeating } \\
\left(10^{-3} \mu \text { moles }\right)\end{array}$ & $\begin{array}{c}\text { Percent } \\
\text { permeating }\end{array}$ & $\begin{array}{c}\text { Permeability } \\
\text { coefficient } \\
\left(10^{-3} \mathrm{~cm}^{-3} \mathrm{~h}^{-1}\right)\end{array}$ \\
\hline Digitoxin (6) & PE present & 1.11 & $0.26 \pm 0.10$ & $2.5 \pm 1.0^{1}$ & $0.16 \pm 0.06^{1}$ \\
Digitoxin (4) & PE absent & 0.45 & $0.14 \pm 0.10$ & $3.3 \pm 2.4^{1}$ & $0.22 \pm 0.16^{1}$ \\
Ouabain (7) & PE present & 3.62 & $6.2 \pm 1.0$ & $18.2 \pm 3.4^{1}$ & $1.15 \pm 0.21^{1}$ \\
Ouabain (6) & PE absent & 2.20 & $11.0 \pm 2.0$ & $51.5 \pm 12.0^{2}$ & $3.19 \pm 0.64^{2}$ \\
Xanthotoxin (7) & PE present & 0.83 & $2.5 \pm 0.3$ & $31.3 \pm 3.9^{1}$ & $1.97 \pm 0.24^{1}$ \\
Xanthotoxin (5) & PE absent & 0.41 & $1.6 \pm 0.2$ & $40.5 \pm 5.5^{1}$ & $3.08 \pm 0.58^{1}$ \\
\hline
\end{tabular}

$*$ Data reported as mean \pm SE. Summary statistics within compounds followed by different numbers are significantly different $(P=0.01)$.

${ }^{a}$ Midgut preparations with a peritrophic envelope present were also surrounded by a fenestrated gut wall. Midgut preparations without a peritrophic envelope had an intact gut wall. 
TABLE 2. Extractive Ultrafiltration (30,000 MWCO) of Digitoxin, Ouabain, and Xanthotoxin in Lysolecithin Suspensions*

\begin{tabular}{lccc}
\hline & \multicolumn{2}{c}{ Concentration $(\mu \mathrm{M})$} & \multicolumn{2}{c}{$\begin{array}{c}\text { Permeability coefficient } \\
\left(10^{-3} \mathrm{~cm}^{-3} \mathrm{~h}^{-1}\right)\end{array}$} \\
\cline { 2 - 4 } Compound $^{\mathrm{a}}$ & Original solution & Ultrafiltrate solution & $0.044 \pm 0.02$ \\
Lysolecithin & 1193.4 & $25.2 \pm 2.2$ & $0.15 \pm 0.005$ \\
Digitoxin & 54.6 & $7.5 \pm 0.3$ & $1.60 \pm 0.10$ \\
Ouabain & 24.5 & $29.5 \pm 0.2$ & $1.00 \pm 0.07$ \\
Xanthotoxin & 190.3 & $120.4 \pm 6.9$ & \\
\hline
\end{tabular}

*Data reported as mean $\pm \mathrm{SE}$.

${ }^{a}$ Suspension of digitoxin, ouabain and xanthotoxin were prepared by sonicating each chemical in a suspension of soy lysolecithin $(1 \mathrm{mg} / \mathrm{ml})$, followed by centrifugation $(13,600 \mathrm{~g})$ and filtration $(0.45 \mu \mathrm{m})$.

1992), and possibly also $S$. gregaria (Scudder and Meredith, 1982), appears to be the result of the combined low permeability of the peritrophic envelope and the midgut wall.

The results of previous work on S. gregaria showing that ouabain is not absorbed from the midgut (Scudder and Meredith, 1982) are in marked contrast to the results on $M$. sanguinipes in this study. Although the permeability of ouabain through $M$. sanguinipes gut preparations is significantly reduced by the presence of the peritrophic envelope, the permeability coefficient for ouabain remains relatively high. Indeed, the percent of ouabain permeating midgut preparations $(18 \%)$ is similar to that for highly permeable compounds such as gallic acid (23\%) (Barbehenn and Martin, 1992). Non-absorption of ouabain is most likely the result of an efficient detoxification system in the midgut epithelium, which is not necessarily fully functional in midgut preparations during the $1.5 \mathrm{~h}$ incubation period. It is also possible that detoxification systems are overwhelmed by the high concentration of ouabain injected into the midgut lumen in this study.

Xanthotoxin permeated complete midgut preparations more rapidly than did the amphiphilic cardiac glycosides, digitoxin and ouabain $(P<0.02)$. This was the opposite of the result expected if xanthotoxin in micellar suspensions is sequestered in the lipophilic cores of lysolecithin micelles (Kuksis, 1987). However, the results of ultrafiltration and particle size studies demonstrated that a large fraction of xanthotoxin does not aggregate with lysolecithin but instead forms small $(1 \mathrm{~nm})$ micelles. Such small micelles (stacked arrangements) are formed by many hydrophobic polycyclic compounds in aqueous solutions (Atwood, 1983). Results on xanthotoxin permeability presented in this study also differ from previous work, which demonstrated nonabsorption of xanthotoxin by $M$. sanguinipes (Berenbaum and Isman, 1989). Extensive work has shown that adapted insects chemically transform xanthotoxin in the midgut epithelium (Ivie et al., 1983; Bull et al., 1984; Nitao, 1989), whereas xanthotoxin permeates the peritrophic envelope and midgut wall in non-adapted insect species (Ivie et al., 1983). In vivo results on midgut wall permeability may differ from the results in this study if midgut detoxification systems in this study were not fully functional or were overwhelmed by a large dose of xanthotoxin. Further work is needed to examine whether lipophilic allelochemicals in general have such high peritrophic envelope permeabilities as does xanthotoxin, or whether this is an attribute of molecules that form small micelles.

The extent to which the molecular weights of the allelochemicals tested might explain their relative permeabilities was examined by estimating their diffusion rates. A regression equation was calculated for protein diffusion coefficients (D) and molecular weights (13.4-524 kDa) (Lehninger, 1975), and for FITC-dextran flux from midgut preparations and molecular weights (20$756 \mathrm{kDa}$ ) (Barbehenn and Martin, 1995). These equations $(\mathrm{D}=-2.56 \times \ln \mathrm{MW}+35.40$ and Flux $=-270 \times \ln \mathrm{MW}+3,620$, respectively) provide surprisingly similar predictions for the relative diffusion rates and fluxes of the allelochemicals tested. The diffusion coefficient of free digitoxin (MW 765) is 96\% of that of ouabain (MW 585), the same as predicted for their relative fluxes through the peritrophic envelope. By contrast, digitoxin aggregated in lysolecithin micelles (MW 92,000 ) would have a diffusion coefficient or flux 
that is $29-34 \%$ of that of free digitoxin or ouabain. In this study the permeability coefficient for digitoxin from gut preparations containing a peritrophic envelope was only $14 \%$ of that of ouabain. These results suggest that digitoxin is aggregated in mixed micelles in the gut lumen in $M$. sanguinipes, and that these lipid aggregates are largely retained in the endoperitrophic space as a result of their low diffusion rates and extractive ultrafiltration by the peritrophic envelope.

\section{ACKNOWLEDGMENTS}

Special thanks to Michael M. Martin for improvements made to the manuscript and to Jim Fete and the Coulter Corporation for the use of a Coulter N4 Plus submicron particle size analyzer. This work was supported by NSF grants IBN-9407128 to M.M.M. and R.V.B and IBN-96-03621 to R.V.B. and M.M.M.

\section{LITERATURE CITED}

Atwood D. 1983. Colloidal properties of drugs. In: Wyn-Jones E, Gormally J, editors. Aggregation processes in solution. New York: Elsevier, p 211-240.

Barbehenn RV, Martin MM. 1992. Protective role of the peritrophic membrane in the tannin-tolerant larvae of Orgyia levcostigma (Lymantriidae). J Insect Physiol 38:973-980.

Barbehenn RV, Martin MM. 1995. Peritrophic envelope permeability in herbivorous insects. J Insect Physiol 41:303-311.

Barbehenn RV, Martin MM, Hagerman AE. 1996. Reassessment of the roles of the peritrophic envelope and hydrolysis in protecting polyphagous grasshoppers from ingested hydrolyzable tannins. J Chem Ecol 22:1901-1919.

Barbehenn RV, Martin MM. 1997. Permeability of the peritrophic envelopes of herbivorous insects to dextran sulfate: a test of the polyanion exclusion hypothesis. J Insect Physiol 43:243-249.

Barbehenn RV, Martin MM. 1998. Formation of insoluble and colloidally dispersed tannic acid complexes in the midgut fluid of Manduca sexta (Lepidoptera: Sphingidae): an explanation for the failure of tannic acid to cross the peritrophic envelopes of lepidopteran larvae. Arch Insect Biochem Physiol 39:109-117.

Berenbaum MR, Isman MB. 1989. Herbivory in holometabolous and hemimetabolous insects: contrasts between Orthoptera and Lepidoptera. Experientia 45:229-236.

Bull DL, Ivie GW, Beier RC, Pryor NW, Oertli EH. 1984.
Fate of photosensitizing furanocoumarins in tolerant and sensitive insects. J Chem Ecol 10:893-911.

Duffey SS. 1980. Sequestration of plant natural products by insects. Annu Rev Entomol 25:447-477.

Ferreira C, Capella AN, Sitnik R, Terra WR. 1994. Properties of the digestive enzymes and the permeability of the peritrophic membrane of Spodoptera frugiperda (Lepidoptera) larvae. Comp Biochem Physiol 107:631-640.

Florence AT. 1977. Biological implications of micelle formation. In: Mittal KL, editor. Micellization, solubilization, and microemulsions. New York: Plenum, p 55-74.

Furth AJ, Bolton H, Potter J, Priddle JD. 1984. Separating detergent from proteins. In: Prokoby WB, editor. Methods in enzymology. New York: Academic, vol 104, p $318-328$.

Hawke JC. 1973. Lipids. In: Butler GW, Bailey RW, editors. Chemistry and biochemistry of herbage. New York: Academic, p 213-263.

Isman MB. 1985) Toxicity and tolerance of sesquiterpene lactones in the migratory grasshopper, Melanoplus sanguinipes (Acrididae). Pesticide Biochem Physiol 24:348-354.

Ivie GW, Bull DL, Beier RC, Pryor NW, Oertli EH. 1983. Metabolic detoxification: mechanism of insect resistance to plant psoralens. Science 221:374-376.

Jones OT, Earnest JP, McNamee MG. 1987. Solubilization and reconstitution of membrane proteins. In: Findlay J, Evans WH, editors. Biological membranes: a practical approach. Washington, DC: IRL Press, p 139-177.

Kuksis A. 1987. Digestion and absorption of fat-soluble xenobiotics. In: A. Kuksis, editor. Fat absorption. CRC Press, Boca Raton, vol. 2, p 87-116.

Lehninger AL. 1975. Biochemistry. New York: Worth.

Lindroth RL. 1991. Differential toxicity of plant allelochemicals to insects: roles of enzymatic detoxication systems. In: Bernays EA, editor. Insect-plant interactions. CRC Press, Boca Raton, vol 3, p 1-33.

Martin MM, Martin JS. 1984. Surfactants: their role in preventing the precipitation of proteins by tannins in insect guts. Oecologia 61:342-345.

Masoro EJ. 1968. Physiological chemistry of lipids in mammals. Philadelphia: WB Saunders.

Merck. 1996. The Merck index. Budavari S, editor. Whitehouse Station, NJ: Merck and Co.

Miller N, Lehane MJ. 1990. In vitro perfusion studies on the peritrophic membrane of the tsetse fly Glossina morsitans morsitans (Diptera, Glossinidae). J Insect Physiol 36:813-818.

Miller N, Lehane MJ. 1993. Ionic environment and the per- 
meability properties of the peritrophic membrane of Glossina morsitans morsitans. J Insect Physiol 39: 139-144.

Mimms LT, Zampighi G, Nozaki Y, Tanford C, Reynolds JA. 1981. Phospholipid vesicle formation and transmembrane incorporation using octyl glucoside. Biochemistry 20:833-840.

Nitao JK. 1989. Enzymatic adaptation in a specialist herbivore for feeding on furanocoumarin-containing plants. Ecology 70:629-635.

Peters W, Wiese B. 1986. Permeability of the peritrophic membranes of some Diptera to labelled dextrans. J Insect Physiol 32:43-49.

Santos CD, Terra WR. 1986. Distribution and characterization of oligomeric digestive enzymes from Erinnyis ello larvae and inferences concerning secretory mechanisms and the permeability of the peritrophic membrane. Insect Biochem 16:691-700.

Scudder GGE, Meredith J. 1982. The permeability of the midgut of three insects to cardiac glycosides. J Insect Physiol 28:689-694.

Shah AH, Guthrie FE. 1970. Penetration of insecticides through the isolated midgut of insects and mammals. Comp Gen Pharmacol 1:391-399.
Small DM. 1986. The physical chemistry of lipids. New York: Plenum.

Smirle MJ, Isman MB. 1992. Metabolism and elimination of ingested allelochemicals in a holometabolous and a hemimetabolous insect. Entomol Exp Appl 62:183-190.

Terra WR, Ferreira C. 1981. The physiological role of the peritrophic membrane and trehalase: digestive enzymes in the midgut and excreta of starved larvae of Rhynchosciara. J Insect Physiol 27:325-331.

Turunen S, Chippendale GM. 1989. Relationship between dietary lipids, midgut lipids, and lipid absorption in eight species of Lepidoptera reared on artificial and natural diets. J Insect Physiol 35:627-633.

Vander AJ, Sherman JH, Luciano DS. 1980. Human physiology. New York: McGraw-Hill.

Watters JC, Miller DA, Le Trac T, Murrer DG. 1989. Extractive ultrafiltration. In: Butterfield DA, editor. Biological and synthetic membranes. New York: Alan R. Liss, pp 181-191.

Wilkinson L. 1990. SYSTAT: The system for statistics. Evanston, IL: SYSTAT.

Zhuzhikov DP. 1970. Permeability of the peritrophic membrane in the larvae of Aedes aegypti. J Insect Physiol 16:1193-1202. 